\title{
Fractional biclique covers and partitions of graphs
}

\author{
Valerie L. Watts \\ Department of Mathematics \\ Malaspina University-College \\ 900 Fifth Street \\ Nanaimo, British Columbia, Canada V9R 5S5 \\ wattsv@mala.bc.ca
}

Submitted: Jun 20, 2005; Accepted: Aug 8, 2006; Published: Aug 22, 2006

Mathematics Subject Classifications: 05C99, 90C35

\begin{abstract}
A biclique is a complete bipartite subgraph of a graph. This paper investigates the fractional biclique cover number, $b c^{*}(G)$, and the fractional biclique partition number, $b p^{*}(G)$, of a graph $G$. It is observed that $b c^{*}(G)$ and $b p^{*}(G)$ provide lower bounds on the biclique cover and partition numbers respectively, and conditions for equality are given. It is also shown that $b c^{*}(G)$ is a better lower bound on the Boolean rank of a binary matrix than the maximum number of isolated ones of the matrix. In addition, it is noted that $b c^{*}(G) \leq b p^{*}(G) \leq \beta^{*}(G)$, the fractional vertex cover number. Finally, the application of $b c^{*}(G)$ and $b p^{*}(G)$ to two different weak products is discussed.
\end{abstract}

Keywords: fractional biclique covers, fractional biclique partitions, Boolean rank, weak products

\section{Introduction}

Fractional graph theory is the modification of integer-valued graph parameters to allow them to take on non-integer values. This article investigates the fractional analogues of the minimum number of complete bipartite subgraphs (bicliques) needed to cover or partition the edges of a graph. For more on fractional graph theory and other fractional graph parameters, see Berge [1] or Scheinerman and Ullman [17].

To begin, some definitions are given which are used throughout. A simple graph is denoted by $G$ with vertex set $V(G)=\{1,2, \cdots, n\}$ and edge set $E(G)$. An edge of $G$ is an unordered pair of vertices $\{u, v\}$, usually written $u v$. For general graph theory terminology used throughout, see [2]. A subgraph of $G$ whose edge set forms a complete bipartite graph is called a biclique of $G$. Let $K(R, S)$ denote the biclique of $G$ with edge set $\{i j: i \in R, j \in S\}$ where $R$ and $S$ are disjoint non-empty subsets of vertices of $G$. 
The sets of vertices $R$ and $S$ are called the bipartition of $K(R, S)$. If $|R|=r$ and $|S|=s$, then $K(R, S)$ is said to be a $K_{r, s}$. A biclique $K(R, S)$ is a star centered at vertex $v$ if one of $R$ or $S$ contains only a single vertex $v$. The set of all bicliques of $G$ is denoted $\mathcal{B}(G)$.

A biclique cover of a graph $G$ is a collection $\mathcal{B}$ of bicliques $K\left(X_{i}, Y_{i}\right), 1 \leq i \leq k$, of $G$ whose edge sets cover the edge set of $G$. That is, each edge of $G$ is in at least one of the bicliques in $\mathcal{B}$. The biclique cover number, $b c(G)$, of a graph $G$ is the minimum number of bicliques in a biclique cover of $G$. A biclique partition of a graph $G$ is a collection $\mathcal{B}$ of bicliques $K\left(X_{i}, Y_{i}\right), 1 \leq i \leq k$, of $G$ whose edge sets partition the edge set of $G$. That is, each edge of $G$ is in exactly one of the bicliques in $\mathcal{B}$. The biclique partition number, $b p(G)$, is the minimum number of bicliques in a biclique partition of $G$. For more on biclique covers and partitions, see $[3,8,11,14,15,16]$.

Sections 2 and 3 introduce the fractional biclique cover and partition numbers, respectively, via linear programs that assign weights to either the bicliques or the edges of a graph. It is observed that the fractional biclique cover (resp. partition) number is a lower bound on $b c(G)$ (resp. $b p(G)$ ). In addition, it is shown that the fractional biclique cover number is a better lower bound on Boolean rank than a well known lower bound given by Gregory and Pullman [10]. An example is given which shows that the fractional biclique cover number may be smaller than the fractional biclique partition number. It is well known that for any graph $G, b c(G) \leq b p(G) \leq \beta(G)$, the vertex cover number, and section 4 shows that the fractional analogues of these three numbers share the same relationship. Finally, section 5 discusses the application of the fractional biclique cover and partition numbers to weak product and weak bipartite product. In particular, it is observed that the fractional biclique cover number of a weak bipartite product of bipartite graphs equals the product of their fractional biclique cover numbers. A similar result is true for weak product.

\section{Fractional Biclique Covers}

Another way to view a biclique cover is as a function $w$ that assigns to each biclique $B$ of $G$ either 0 or 1 so that, for each edge $e \in E(G), \sum w(B) \geq 1$ where the sum is taken over all bicliques that contain $e$. Then, $b c(G)$ is the minimum of $\sum_{B \in \mathcal{B}(G)} w(B)$ over all biclique covers. Thus, $b c(G)$ is the value of a $(0,1)$-integer program and its linear relaxation defines the fractional biclique cover number.

A fractional biclique cover is a function $w$ that assigns to each biclique $B$ of a graph $G$ a number so that $w(B) \geq 0$ and, for each edge $e \in E(G), \sum w(B) \geq 1$ where the sum is taken over all bicliques that contain $e$. Note that every biclique cover is in fact a fractional biclique cover. To compare biclique covers and fractional biclique covers, consider the complete graph on four vertices, $K_{4}$, shown in Figure 1. A biclique cover of $K_{4}$ is given by bicliques $B_{1}=K(\{1,3\},\{2,4\})$ and $B_{2}=K(\{1,2\},\{3,4\})$. The function $w$ associated with this cover assigns the values $w\left(B_{1}\right)=w\left(B_{2}\right)=1$ and 0 for all other bicliques of $K_{4}$. As observed above, $w$ is also a fractional biclique cover. Now, consider the function $w_{1}$ where $w_{1}(K(\{1,3\},\{2,4\}))=w_{1}(K(\{1,2\},\{3,4\}))=$ 
$w_{1}(K(\{1,4\},\{2,3\}))=\frac{1}{2}$ and $w_{1}(B)=0$ for all other bicliques $B$ of $K_{4}$. Note that $w_{1}$ is a fractional biclique cover of $K_{4}$ since each edge $e$ of $K_{4}$ is in exactly two of the bicliques $K(\{1,3\},\{2,4\}), K(\{1,2\},\{3,4\}), K(\{1,4\},\{2,3\})$ and so $\sum_{\{B: e \in B\}} w_{1}(B) \geq 1$.

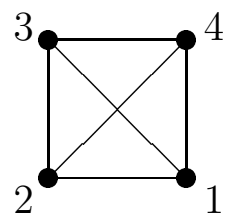

\section{Figure 1}

The fractional biclique cover number, $b c^{*}(G)$, is the infimum of $\sum_{B \in \mathcal{B}(G)} w(B)$ over all fractional biclique covers. That is, $b c^{*}(G)$ is the value of the linear program

$$
\begin{array}{ll}
\text { minimize } & \sum_{B \in \mathcal{B}(G)} w(B) \\
\text { subject to } & \sum_{\{B: e \in B\}} w(B) \geq 1 \text { for each edge } e \text { of } G \\
& w(B) \geq 0 \text { for each biclique } B \text { of } G
\end{array}
$$

where each biclique $B$ of $G$ is assigned a weight $w(B)$. Since the value of the fractional biclique cover number is determined by a linear program, $b c^{*}(G)$ may also be found using the dual program. Thus, $b c^{*}(G)$ is the value of the linear program

$$
\begin{array}{ll}
\text { maximize } & \sum_{e \in E(G)} v(e) \\
\text { subject to } & \sum_{e \in B} v(e) \leq 1 \text { for each biclique } B \text { of } G \\
& v(e) \geq 0 \text { for each edge } e \text { of } G
\end{array}
$$

where each edge $e$ of $G$ is assigned a weight $v(e)$. Note that $v(e) \leq 1$ since each edge is a biclique. For results regarding linear programming mentioned throughout, see Chvátal [4].

An automorphism of $G$ is a permutation of the vertices of $G$ which maps edges to edges and non-edges to non-edges. Let Aut $G$ denote the automorphism group of a graph $G$. The orbits of Aut $G$ partition the edge set of $G$ into equivalence classes. It is straightforward to check that if $v$ is an optimal weighting of the edges of $G$ in $(2)$ with $b c^{*}(G)=\sum_{e \in E(G)} v(e)$ and $\hat{v}(e)=\frac{1}{|\operatorname{Aut} G|} \sum_{\sigma \in \operatorname{Aut} G} v(\sigma(e))$ then $\hat{v}(e)$ also satisfies the constraints of (2) and $b c^{*}(G)=\sum_{e \in E(G)} \hat{v}(e)$. Thus, in finding $b c^{*}(G)$ using (2), edges in the same orbit of Aut $G$ may be assumed to have the same weight. In particular, if $G$ is edge-transitive (that is, if for each pair of edges $e$ and $f$ of $G$ there exists $\sigma \in$ Aut $G$ with $\sigma(e)=f$ ) then each edge of $G$ may be assumed to have the same weight. Consequently, $b c^{*}(G)=\frac{|E(G)|}{a b}$ where $K_{a, b}$ is the largest biclique of an edge-transitive graph $G$ in terms of the number of edges. For the proofs of these statements, see Watts [19].

The cycle $C_{n}$ on $n$ vertices is an edge-transitive graph and for $n \neq 4$ its largest biclique is a $K_{1,2}$. Thus, $b c^{*}\left(C_{n}\right)=\frac{n}{2}$ for $n \neq 4$ and $b c^{*}\left(C_{4}\right)=1$. Similarly, the complete graph $K_{n}$ on $n$ vertices is edge-transitive and its largest biclique is a $K_{\left\lceil\frac{n}{2}\right\rceil,\left\lfloor\frac{n}{2}\right\rfloor}$. Consequently,

$$
b c^{*}\left(K_{n}\right)= \begin{cases}\frac{2(n-1)}{n} & \text { if } n \text { is even } \\ \frac{2 n^{n}}{n+1} & \text { if } n \text { is odd }\end{cases}
$$


Harary, Hsu and Miller [12] showed that $b c\left(K_{n}\right)=\left\lceil\log _{2} n\right\rceil$ and so as $n \rightarrow \infty, b c\left(K_{n}\right) \rightarrow \infty$ while $b c^{*}\left(K_{n}\right) \rightarrow 2$.

Consider the complement of the cycle on six vertices, $\bar{C}_{6}$, shown in Figure 2 below. The orbits of Aut $\bar{C}_{6}$ yield the following equivalence classes: $A=\{13,15,24,26,35,46\}$ and $B=\{14,25,36\}$. According to the statement given above, the edges in $A$ may all receive the same weight, $a$, and the edges in $B$ may all receive the same weight, $b$. It follows that the objective function of (2) for $\bar{C}_{6}$ is $6 a+3 b$ since there are six elements in $A$ and three elements in $B$. The seven different stars centered at vertex 1 produce the following constraints:

\begin{tabular}{|c|c|}
\hline Biclique & Constraint \\
\hline$K(\{1\},\{3\})$ & $a \leq 1$ \\
$K(\{1\},\{4\})$ & $b \leq 1$ \\
$K(\{1\},\{5\})$ & $a \leq 1$ \\
$K(\{1\},\{3,5\})$ & $2 a \leq 1$ \\
$K(\{1\},\{3,4\})$ & $a+b \leq 1$ \\
$K(\{1\},\{4,5\})$ & $a+b \leq 1$ \\
$K(\{1\},\{3,4,5\})$ & $2 a+b \leq 1$ \\
\hline
\end{tabular}

Similar constraints occur for the stars centered at each vertex $i$. The only other bicliques in $\bar{C}_{6}$ are $K_{2,2}$ 's, such as $K(\{1,2\},\{4,5\})$. Each $K_{2,2}$ yields the same constraint: $2 a+2 b \leq 1$. Then from $(2), b c^{*}\left(\bar{C}_{6}\right)$ is the value of the linear program

$$
\begin{array}{ll}
\text { maximize } & 6 a+3 b \\
\text { subject to } & 2 a \leq 1 \\
& a+b \leq 1 \\
& 2 a+b \leq 1 \\
& 2 a+2 b \leq 1 \\
& a, b \leq 1
\end{array}
$$

Since $2 a+b \leq 1$, it follows that $6 a+3 b \leq 3$. Taking $a=\frac{1}{2}$ and $b=0$, the maximum of 3 may be attained. Therefore, $b c^{*}\left(\bar{C}_{6}\right)=3$.

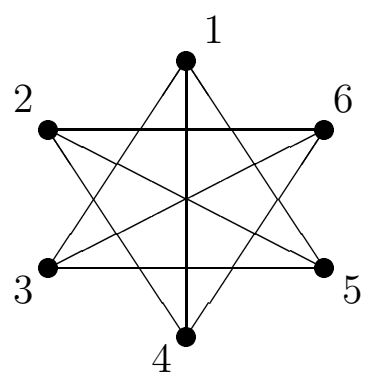

\section{Figure 2}

Theorem 2.1 shows that the fractional biclique cover number is a lower bound for the biclique cover number and conditions for equality are given. The proof of Theorem 2.1 first appeared in the thesis of the author [19]. 
Theorem 2.1 For a graph $G, b c^{*}(G) \leq b c(G)$ with equality holding if and only if for some (and every) minimum biclique cover $\mathcal{B}$ and optimal weighting $v$ of the edges of $G$ for (2), $\sum_{e \in B} v(e)=1$ for all $B \in \mathcal{B}, \sum_{e \in B} v(e) \leq 1$ for all bicliques $B$ of $G, v(e)=0$ for each edge e of $G$ that is covered more than once by the bicliques of $\mathcal{B}$ and $v(e) \geq 0$ for each edge e of $G$.

Proof. Let $\mathcal{B}$ be a minimum biclique cover of $G$. Let $v$ be an optimal weighting of the edges of $G$ for $(2)$. Then $b c^{*}(G)=\sum_{e \in E(G)} v(e) \leq \sum_{B \in \mathcal{B}}\left(\sum_{e \in B} v(e)\right) \leq \sum_{B \in \mathcal{B}} 1=|\mathcal{B}|=$ $b c(G)$. Thus, $b c^{*}(G)=b c(G)$ if and only if $\sum_{e \in B} v(e)=1$ for all $B \in \mathcal{B}$ and $v(e)=0$ for each edge $e$ of $G$ which is covered more than once by the bicliques of $\mathcal{B}$.

The case of bipartite graphs is of special interest since $b c^{*}(G)$ provides a lower bound on Boolean rank. The Boolean rank, $r_{B}(A)$, of an $m \times n$ Boolean matrix $A$ is the smallest integer $k$ such that $A=X Y^{T}$ for some $m \times k$ binary matrix $X$ and $n \times k$ binary matrix $Y$. If $A$ is an $m \times n(0,1)$-matrix and $G$ is the bipartite graph with bipartite adjacency matrix $A$, then $b c(G)=r_{B}(A)$, an observation provided by Orlin [16]. Consequently, $b c^{*}(G)$ is the fractional analogue of $r_{B}(A)$ and $r_{B}(A) \geq b c^{*}(G)$. For more on Boolean rank, see $[6,7,8,10,13,15]$

As in [10], a set of ones of a binary matrix $A$ is isolated if no pair of ones are in an all-ones submatrix of $A$ together. Let $i(A)$ be the maximum number of ones in an isolated set of $A$. It follows that $r_{B}(A) \geq i(A)$. However, Theorem 2.2 below implies that $b c^{*}(G) \geq i(A)$ where $G$ is the bipartite graph with bipartite adjacency matrix $A$. Consequently, fractional Boolean rank is a better lower bound for $r_{B}(A)$ than $i(A)$. For

example, the $5 \times 5$ matrix $\bar{I}_{5}$ with zeros down the main diagonal and ones everywhere else has $r_{B}\left(\bar{I}_{5}\right)=4, r_{B}^{*}\left(\bar{I}_{5}\right)=\frac{10}{3}$ and $i\left(\bar{I}_{5}\right)=3$.

Theorem 2.2 For a bipartite graph $G, b c^{*}(G) \geq i(A)$, where $A$ is the bipartite adjacency matrix of $G$.

Proof. A set of isolated ones in $A$ corresponds to a matching in $G$ with the property that the subgraph induced by the matching is $K_{2,2}$-free. This implies that the edges of the matching cannot be in any biclique together. Let $M$ be the matching corresponding to a maximum isolated set of $A$. Define a weighting $v$ on the edges of $G$ as follows:

$$
v(e)= \begin{cases}1 & \text { if } e \in M \\ 0 & \text { otherwise }\end{cases}
$$

This weighting of the edges of $G$ satisfies the constraints of (2) since, for any biclique $B$ of $G, \sum_{e \in B} v(e) \leq 1$. Thus, $b c^{*}(G) \geq \sum_{e \in E(G)} v(e)=|M|=i(A)$.

\section{$3 \quad$ Fractional Biclique Partitions}

A biclique partition is a function $w$ that assigns each biclique $B$ of $G$ either 0 or 1 so that, for each edge $e \in E(G), \sum w(B)=1$ where the sum is taken over all bicliques 
that contain $e$. Then, $b p(G)$ is the minimum of $\sum_{B \in \mathcal{B}(G)} w(B)$ over all biclique partitions. Thus, $b p(G)$ is the value of a $(0,1)$-integer program and its linear relaxation defines the fractional biclique partition number.

A fractional biclique partition is a function $w$ that assigns to each biclique $B$ of a graph $G$ a number so that $w(B) \geq 0$ and, for each edge $e \in E(G), \sum w(B)=1$ where the sum is taken over all bicliques that contain $e$. The fractional biclique partition number, $b p^{*}(G)$, is the infimum of $\sum_{B \in \mathcal{B}(G)} w(B)$ over all fractional biclique partitions. That is, $b p^{*}(G)$ is the value of the linear program

$$
\begin{array}{ll}
\text { minimize } & \sum_{B \in \mathcal{B}(G)} w(B) \\
\text { subject to } & \sum_{\{B: e \in B\}} w(B)=1 \text { for each edge } e \text { of } G \\
& w(B) \geq 0 \text { for each biclique } B \text { of } G
\end{array}
$$

where each biclique $B$ of $G$ is assigned a weight $w(B)$. As before, the dual program may also be used. Thus, $b p^{*}(G)$ is the value of the linear program

$$
\begin{array}{ll}
\text { maximize } & \sum_{e \in E(G)} v(e) \\
\text { subject to } & \sum_{e \in B} v(e) \leq 1 \text { for each biclique } B \text { of } G
\end{array}
$$

where each edge $e$ of $G$ is assigned a weight $v(e)$. Note that in (4) edges may receive negative weights, whereas in (2) non-negative weights are required. Similar to the case with $b c^{*}(G)$, edges in the same orbit of Aut $G$ may receive the same weight when (4) is used to determine $b p^{*}(G)$.

In the examples immediately before Theorem 2.1, each optimal weighting of the edges for (2) is also an optimal weighting of the edges for (4). Consequently, $b c^{*}\left(C_{n}\right)=b p^{*}\left(C_{n}\right)$, $b c^{*}\left(K_{n}\right)=b p^{*}\left(K_{n}\right)$ and $b c^{*}\left(\bar{C}_{6}\right)=b p^{*}\left(\bar{C}_{6}\right)$. As with the fractional biclique cover number, the fractional biclique partition number is a lower bound on the biclique partition number. The proof of Theorem 3.1 is similar to the proof of Theorem 2.1 and is omitted. Alternatively, the proof may be found in Watts [19].

Theorem 3.1 For a graph $G, b p^{*}(G) \leq b p(G)$ with equality holding if and only if for some (and every) minimum biclique partition $\mathcal{B}$ and optimal weighting $v$ of the edges of $G$ for (4), $\sum_{e \in B} v(e)=1$ for all $B \in \mathcal{B}$ and $\sum_{e \in B} v(e) \leq 1$ for all bicliques $B$ of $G$.

A well-known lower bound on $b p(G)$ is the eigenvalue bound, attributed to H.S. Witsenhausen by Graham and Pollak [9], which states that $b p(G) \geq \max \left\{n_{+}(G), n_{-}(G)\right\}$, where $n_{+}(G)$ (resp. $n_{-}(G)$ ) is the number of positive (resp. negative) eigenvalues of the adjacency matrix of $G$. Although Theorem 3.1 provides a lower bound for $b p(G)$, in general $b p^{*}(G)$ is not as good a lower bound on $b p(G)$ as $\max \left\{n_{+}(G), n_{-}(G)\right\}$. Theorem 3.2 below shows that $b p^{*}(G) \leq \frac{n}{2}$, the proof of which first appeared in the thesis of the author [19]. Consequently, the only instances when $b p^{*}(G)$ may be of interest as a lower bound on $b p(G)$ is when $\max \left\{n_{+}(G), n_{-}(G)\right\} \leq \frac{n}{2}$. An example of this is $\bar{C}_{6}$ since it was observed above that $b p^{*}\left(\bar{C}_{6}\right)=b p\left(\bar{C}_{6}\right)=3$ but $\max \left\{n_{+}\left(\bar{C}_{6}\right), n_{-}\left(\bar{C}_{6}\right)\right\}=2$. Other graphs which have $\max \left\{n_{+}(G), n_{-}(G)\right\} \leq \frac{n}{2}$ include all the bipartite graphs. 
Theorem 3.2 For a graph $G$ with $n$ vertices, $b p^{*}(G) \leq \frac{n}{2}$.

Proof. Let $v$ be an optimal weighting of the edges of $G$ for (4). For each vertex $x$ in $G$, let $S_{x}$ be the star centered at $x$, containing all the edges incident to $x$. Then $b p^{*}(G)=\sum_{e \in E(G)} v(e)=\frac{1}{2} \sum_{x \in V(G)}\left(\sum_{e \in S_{x}} v(e)\right) \leq \frac{1}{2} \sum_{x \in V(G)} 1=\frac{n}{2}$.

As with the fractional biclique cover number, the bipartite case is of special interest. Not only are bipartite graphs of interest because of the eigenvalue bound mentioned above, but also because the fractional biclique partition number gives a lower bound on the non-negative integer rank of a binary matrix . The non-negative integer rank, $r_{\mathbb{Z}^{+}}(A)$, of an $m \times n$ matrix $A$ with non-negative integer entries is the smallest integer $k$ such that $A=X Y^{T}$ for some $m \times k$ matrix $X$ and $n \times k$ matrix $Y$, both with non-negative integer entries. If $A$ is an $m \times n(0,1)$-matrix and $G$ is the bipartite graph with bipartite adjacency matrix $A$, then $b p(G)=r_{\mathbb{Z}^{+}}(A)$, an observation provided by Orlin [16]. Consequently, $b p^{*}(G)$ is the fractional analogue of $r_{\mathbb{Z}^{+}}(A)$ and $r_{\mathbb{Z}^{+}}(A) \geq b p^{*}(G)$ when $G$ is bipartite. For more on non-negative integer rank, see $[5,8,15]$.

It is always interesting to see if known integer results have corresponding fractional analogues. Since every biclique partition is a biclique cover, it follows that $b c(G) \leq b p(G)$. The fractional analogue of this statement is given in Theorem 3.3, together with conditions for equality. The proof of Theorem 3.3 first appeared in Watts [19].

Theorem 3.3 For a graph $G, b c^{*}(G) \leq b p^{*}(G)$ with equality holding if and only if for some optimal weighting $v$ of the edges of $G$ for $(4), v(e) \geq 0$ for all edges $e$ of $G$.

Proof. It follows immediately from (2) and (4) that $b c^{*}(G) \leq b p^{*}(G)$ since a weighting $v$ of the edges of $G$ that satisfies the constraints of (2) also satisfies the constraints of (4). To prove the characterization for equality, suppose $v$ is an optimal weighting of the edges of $G$ for (4) with $v(e) \geq 0$ for all edges $e$ of $G$. Then $v$ satisfies the constraints of (2) and so $b c^{*}(G) \geq \sum_{e \in E(G)} v(e)=b p^{*}(G)$. It follows that $b c^{*}(G)=b p^{*}(G)$.

Conversely, suppose that $b c^{*}(G)=b p^{*}(G)$ and $v$ is an optimal weighting of the edges of $G$ for (2). Then $v$ satisfies the constraints of $(4)$ and $b c^{*}(G)=\sum_{e \in E(G)} v(e)=b p^{*}(G)$. Thus $v$ must be an optimal weighting of the edges of $G$ for $(4)$ with $v(e) \geq 0$ for each edge $e$ of $G$.

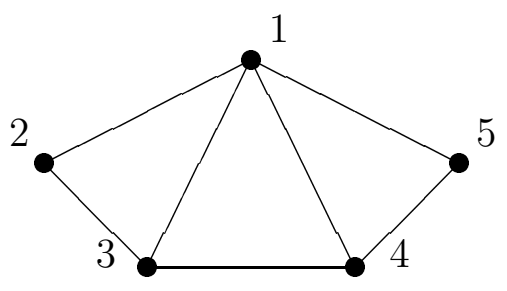

\section{Figure 3}

Theorem 3.3 implies that to have a graph with $b c^{*}(G) \neq b p^{*}(G)$, an optimal weighting of the edges of $G$ for (4) must have some edges that receive negative weights. The graph 
$G$ shown in Figure 3 above is an example of a graph with $b c^{*}(G) \neq b p^{*}(G)$. Under the automorphism group, the orbits of Aut $G$ yield the following equivalence classes: $X_{1}=\{12,15\}, X_{2}=\{13,14\}, X_{3}=\{23,45\}, X_{4}=\{34\}$. Assign each edge in $X_{i}$ weight $x_{i}$ for $1 \leq i \leq 4$. Then, according to $(4), b p^{*}(G)$ is the value of the linear program

$$
\begin{array}{cl}
\operatorname{maximize} & 2 x_{1}+2 x_{2}+2 x_{3}+x_{4} \\
\text { subject to } & 2 x_{1}+2 x_{2} \leq 1 \\
& 2 x_{1}+x_{2} \leq 1 \\
& x_{1}+x_{2} \leq 1 \\
& 2 x_{1} \leq 1 \\
& 2 x_{2} \leq 1 \\
& x_{1}+2 x_{2} \leq 1 \\
& x_{1}+x_{3} \leq 1 \\
& x_{2}+x_{3}+x_{4} \leq 1 \\
& x_{2}+x_{3} \leq 1 \\
& x_{3}+x_{4} \leq 1 \\
& x_{2}+x_{4} \leq 1 \\
& x_{1}+x_{2}+x_{3}+x_{4} \leq 1 \\
& x_{1}, x_{2}, x_{3}, x_{4} \leq 1
\end{array}
$$

Solving the linear program (5) yields $b p^{*}(G)=\frac{9}{4}$ with $x_{1}=x_{2}=\frac{1}{4}, x_{3}=\frac{3}{4}$ and $x_{4}=-\frac{1}{4}$. Adding the additional constraints $x_{1}, x_{2}, x_{3}, x_{4} \geq 0$ to (5) gives the linear program for $b c^{*}(G)$ in $(2)$. Solving this new linear program gives $b c^{*}(G)=2$ with $x_{1}=x_{3}=\frac{1}{2}$ and $x_{2}=x_{4}=0$.

\section{Fractional Vertex Covers}

The vertex cover number, $\beta(G)$, of a graph $G$ is the minimum number of vertices in a vertex cover of $G$. As described in Berge [1], a fractional vertex cover is a function $g$ that assigns to each vertex $v$ of $G$ a number so that $0 \leq g(v) \leq 1$ and for each edge $e \in E(G)$, $\sum g(v) \geq 1$ where the sum is taken over all vertices incident to $e$. The fractional vertex cover number, $\beta^{*}(G)$, is the infimum of $\sum_{v \in V(G)} g(v)$ over all fractional vertex covers. That is, $\beta^{*}(G)$ is the value of the linear program

$$
\begin{array}{ll}
\text { minimize } & \sum_{v \in V(G)} g(v) \\
\text { subject to } & \sum_{e \in v} g(v) \geq 1 \text { for each edge } e \text { of } G \\
& 0 \leq g(v) \leq 1 \text { for each vertex } v \text { of } G
\end{array}
$$

where each vertex $v$ of $G$ is assigned a weight $g(v)$. Dually, $\beta^{*}(G)$ is the value of the linear program

$$
\begin{array}{ll}
\text { maximize } & \sum_{e \in E(G)} f(e) \\
\text { subject to } & \sum_{\{e: v \in e\}} f(e) \leq 1 \text { for each vertex } v \text { of } G \\
& 0 \leq f(e) \leq 1 \text { for each edge } e \text { of } G
\end{array}
$$


where each edge $e$ of $G$ is assigned a weight $f(e)$. In fact, the dual program given in (7) is the linear program for the fractional matching number of $G$. For more on fractional vertex covers and fractional matchings, see Berge [1] or Scheinerman and Ullman [17].

A biclique partition may always be obtained by successively deleting the edge sets of stars centered at the vertices in a vertex cover of $G$. This gives a biclique partition of $G$ consisting entirely of stars and consequently $b c(G) \leq b p(G) \leq \beta(G)$. It follows immediately from $(2)$ and $(7)$ that $b c^{*}(G) \leq \beta^{*}(G)$. In fact, Theorem 4.1 shows that $b c^{*}(G) \leq b p^{*}(G) \leq \beta^{*}(G)$.

Theorem 4.1 For any graph $G, b c^{*}(G) \leq b p^{*}(G) \leq \beta^{*}(G)$.

Proof. It was observed in Theorem 3.3 that $b c^{*}(G) \leq b p^{*}(G)$ and so it remains to prove $b p^{*}(G) \leq \beta^{*}(G)$. Let $v$ be an optimal weighting of the edges of $G$ for (4). Construct a new weighting $f$ for the edges of $G$ as follows:

$$
f(e)=\left\{\begin{array}{cl}
v(e) & \text { if } v(e) \geq 0 \\
0 & \text { if } v(e)<0
\end{array}\right.
$$

Note that for any vertex $x \in V(G)$, the edges incident to $x$ with $v(e) \geq 0$ form a star centered at $x$. Since $v$ satisfies the constraints of $(4), \sum_{v(e) \geq 0} v(e) \leq 1$. Thus, for each $x \in V(G), \sum_{\{e: x \in e\}} f(e)=\sum_{v(e) \geq 0} v(e) \leq 1$. Hence, $f$ satisfies the constraints of $(7)$ and $b p^{*}(G)=\sum_{e \in E(G)} v(e) \leq \sum_{v(e) \geq 0} v(e)=\sum_{e \in E(G)} f(e) \leq \beta^{*}(G)$.

\section{$5 \quad$ Weak Bipartite Products and Weak Products}

Let $\left(X_{G}, Y_{G}\right)$ be the (ordered) bipartition of a bipartite graph $G$. The weak bipartite product of bipartite graphs $G$ and $H$ is the bipartite graph $G \tilde{\times} H$ with ordered bipartition $\left(X_{G} \times X_{H}, Y_{G} \times Y_{H}\right)$. Two vertices $\left(g_{i}, h_{k}\right)$ and $\left(g_{j}, h_{\ell}\right)$ in $G \tilde{\times} H$ are adjacent if and only if $g_{i}$ is adjacent to $g_{j}$ in $G$ and $h_{k}$ is adjacent to $h_{\ell}$ in $H$. In fact, $G \tilde{\times} H$ is one of the components of the weak product $G \times H$ described below. Note that each edge $g_{i} g_{j}$ of $G$ and each edge $h_{k} h_{\ell}$ of $H$ yield only one edge of $G \tilde{\times} H$. Further, every edge of $G \tilde{\times} H$ is the result of a unique pair of edges of $G$ and $H$.

For a bipartite graph $G$ with ordered bipartition $\left(X_{G}, Y_{G}\right)$ each biclique of $G$ may be written as $K(R, S)$ with $R \subseteq X_{G}$ and $S \subseteq Y_{G}$. Then, the biclique $K(R, S)$ is called an ordered biclique of $G$ with ordered bipartition $(R, S)$. All of the bicliques of $G$ may assumed to be ordered. Hence, the bicliques of $G, H$ and $G \tilde{\times} H$ may all be assumed to be ordered bicliques. Note that the weak bipartite product of an ordered biclique $K\left(R_{G}, S_{G}\right)$ of $G$ and an ordered biclique $K\left(R_{H}, S_{H}\right)$ of $H$ yields an ordered biclique $K\left(R_{G}, S_{G}\right) \tilde{\times} K\left(R_{H}, S_{H}\right)=$ $K\left(R_{G} \times R_{H}, S_{G} \times S_{H}\right)$ of $G \tilde{\times} H$ with ordered bipartition $\left(R_{G} \times R_{H}, S_{G} \times S_{H}\right)$. A biclique of $G \tilde{\times} H$ which can be written as the weak bipartite product of an ordered biclique of $G$ and an ordered biclique of $H$ is called an ordered product biclique of $G \tilde{\times} H$. Every ordered product biclique of $G \tilde{\times} H$ can be expressed uniquely as the weak bipartite product of an ordered biclique from $\mathcal{B}(G)$ and an ordered biclique from $\mathcal{B}(H)$. Let $\mathcal{B}(G) \tilde{\times} \mathcal{B}(H)$ denote 
the set of ordered product bicliques of $G \tilde{\times} H$. Then $\mathcal{B}(G) \tilde{\times} \mathcal{B}(H)$ is a subset of all the ordered bicliques of $G \tilde{\times} H$ and $|\mathcal{B}(G) \tilde{\times} \mathcal{B}(H)|=|\mathcal{B}(G)||\mathcal{B}(H)|$.

The weak product of two graphs $G$ and $H$, not necessarily bipartite, is the graph $G \times H$ with vertex set $V(G) \times V(H)$. Two vertices $\left(g_{i}, h_{k}\right)$ and $\left(g_{j}, h_{\ell}\right)$ are adjacent in $G \times H$ if and only if $g_{i}$ is adjacent to $g_{j}$ in $G$ and $h_{k}$ is adjacent to $h_{\ell}$ in $H$. Note that each edge $g_{i} g_{j}$ of $G$ and each edge $h_{k} h_{\ell}$ of $H$ yields two edges of $G \times H$ : the edges $\left(g_{i}, h_{k}\right)\left(g_{j}, h_{\ell}\right)$ and $\left(g_{i}, h_{\ell}\right)\left(g_{j}, h_{k}\right)$. Further, every edge of $G \times H$ belongs to a unique pair of this type.

The weak product, $B_{G} \times B_{H}$, of a biclique $B_{G}=K\left(R_{G}, S_{G}\right)$ of $G$ and a biclique $B_{H}=K\left(R_{H}, S_{H}\right)$ of $H$ yields two disjoint bicliques of $G \times H$ : one is $B_{G} \tilde{\times} B_{H}=K\left(R_{G} \times\right.$ $\left.R_{H}, S_{G} \times S_{H}\right)$ defined above and the other is $K\left(R_{G} \times S_{H}, S_{G} \times R_{H}\right)$. A biclique of $G \times H$ which is one of the two bicliques produced from the weak product of a biclique of $G$ and a biclique of $H$ is called a product biclique of $G \times H$. Each product biclique of $G \times H$ belongs to a unique pair of bicliques $B_{G} \times B_{H}$. Let $\mathcal{B}(G) \times \mathcal{B}(H)$ denote the set of all product bicliques of $G \times H$. Then $\mathcal{B}(G) \times \mathcal{B}(H)$ is a subset of all the bicliques of $G \times H$ and $|\mathcal{B}(G) \times \mathcal{B}(H)|=2|\mathcal{B}(G)||\mathcal{B}(H)|$.

Let $A_{G}$ and $A_{H}$ be the bipartite adjacency matrices of bipartite graphs $G$ and $H$ respectively. Then $A_{G} \otimes A_{H}$ is the bipartite adjacency matrix of $G \tilde{\times} H$, where $\otimes$ denotes the Kronecker product of matrices $A_{G}$ and $A_{H}$. A result of de Caen, Gregory and Pullman [7] showed that $r_{\mathbb{Z}^{+}}\left(A_{G} \otimes A_{H}\right) \leq r_{\mathbb{Z}^{+}}\left(A_{G}\right) r_{\mathbb{Z}^{+}}\left(A_{H}\right)$. It follows that $b p(G \tilde{\times} H) \leq b p(G) b p(H)$. Also, Kratzke, Reznick and West [14] observed that $b p(G \times H) \leq 2 b p(G) b p(H)$. Theorem 5.1 gives the corresponding fractional analogues, the proof of which first appeared in the thesis of the author [19]. Note that Theorem 5.1 gives an inequality for the fractional analogue of the non-negative integer rank of the Kronecker product of binary matrices.

Theorem 5.1 1. Let $G_{i}, 1 \leq i \leq k$, be bipartite graphs with ordered bipartitions $\left(X_{G_{i}}, Y_{G_{i}}\right)$ respectively. Then $b p^{*}\left(G_{1} \tilde{\times} \cdots \tilde{\times} G_{k}\right) \leq \prod_{i=1}^{k} b p^{*}\left(G_{i}\right)$.

2. Let $G_{i}, 1 \leq i \leq k$, be graphs. Then $b p^{*}\left(G_{1} \times \cdots \times G_{k}\right) \leq 2^{k-1} \prod_{i=1}^{k} b p^{*}\left(G_{i}\right)$.

Proof. The proof of 1 follows below. The proof of 2 is similar and is omitted. It suffices to prove the result for $k=2$; the general case follows directly by induction on $k$. Let $G=G_{1}$ and $H=G_{2}$. Let $w_{G}$ and $w_{H}$ be optimal weightings of the ordered bicliques of $G$ and $H$, respectively, for (3). Construct a weighting $w$ of the ordered bicliques of $G \tilde{\times} H$ from the ordered bicliques of $G$ and $H$. If $B$ is an ordered product biclique of $G \tilde{\times} H$ and $B=B_{G} \tilde{\times} B_{H}$ where $B_{G} \in \mathcal{B}(G)$ and $B_{H} \in \mathcal{B}(H)$, let $w(B)=w_{G}\left(B_{G}\right) w_{H}\left(B_{H}\right)$. For all other ordered bicliques $B$ of $G \tilde{\times} H$, let $w(B)=0$.

Note that by construction $w(B) \geq 0$ for each ordered biclique $B$ of $G \tilde{\times} H$. Let $e=$ $\left(g_{i}, h_{k}\right)\left(g_{j}, h_{\ell}\right)$ be an edge of $G \tilde{\times} H$ with $\left(g_{i}, h_{k}\right) \in X_{G} \times X_{H}$ and $\left(g_{j}, h_{\ell}\right) \in Y_{G} \times Y_{H}$. Then $g_{i} g_{j}$ and $h_{k} h_{\ell}$ are edges of $G$ and $H$ respectively. Note that $e$ is an edge of an ordered product biclique $B=B_{G} \tilde{\times} B_{H}$ if and only if $g_{i} g_{j}$ and $h_{k} h_{\ell}$ are edges of the ordered bicliques $B_{G}$ of $G$ and $B_{H}$ of $H$ respectively. Then, remembering that at each stage the bicliques are ordered accordingly, for each such edge $e$, 


$$
\begin{aligned}
\sum_{\{B: e \in B\}} w(B)= & \sum_{\substack{\{B: e \in B\} \\
B \in \mathcal{B}(G) \tilde{\times} \mathcal{B}(H)}} w(B) \\
= & \sum_{\substack{\left\{B_{G} \tilde{\times} B_{H}: e \in B_{G} \tilde{\times} B_{H}\right\} \\
B_{G} \in \mathcal{B}(G), B_{H} \in \mathcal{B}(H)}} w_{G}\left(B_{G}\right) w_{H}\left(B_{H}\right) \\
= & \sum_{\substack{\left\{B_{G}: g_{i} g_{j} \in B_{G}\right\} \\
\left\{B_{H}: h_{k} h_{\ell} \in B_{H}\right\}}} w_{G}\left(B_{G}\right) w_{H}\left(B_{H}\right) \\
= & \left(\sum_{\left\{B_{G}: g_{i} g_{j} \in B_{G}\right\}} w_{G}\left(B_{G}\right)\right)\left(\sum_{\left\{B_{H}: h_{k} h_{\ell} \in B_{H}\right\}} w_{H}\left(B_{H}\right)\right) \\
= & 1 .
\end{aligned}
$$

Thus, $w$ satisfies the constraints of (3) and it follows that

$$
\begin{aligned}
b p^{*}(G \tilde{\times} H) & \leq \sum_{B \in \mathcal{B}(G) \tilde{\times} \mathcal{B}(H)} w(B) \\
& =\sum_{\substack{B=B_{G} \tilde{\times} B_{H} \\
B \in \mathcal{B}(G) \times \mathcal{B}(H)}} w_{G}\left(B_{G}\right) w_{H}\left(B_{H}\right) \\
& =\sum_{\substack{B_{G} \in \mathcal{B}(G) \\
B_{H} \in \mathcal{B}(H)}} w_{G}\left(B_{G}\right) w_{H}\left(B_{H}\right) \\
& =\left(\sum_{\substack{B_{G} \in \mathcal{B}(G)\\
}} w_{G}\left(B_{G}\right)\right)\left(\sum_{B_{H} \in \mathcal{B}(H)} w_{H}\left(B_{H}\right)\right) \\
& b p^{*}(G) b p^{*}(H) .
\end{aligned}
$$

A result of de Caen, Gregory and Pullman [7] showed $r_{B}\left(A_{G} \otimes A_{H}\right) \leq r_{B}\left(A_{G}\right) r_{B}\left(A_{H}\right)$ where $A_{G}$ and $A_{H}$ are the bipartite adjacency matrices of bipartite graphs $G$ and $H$ respectively. Consequently, $b c(G \tilde{\times} H) \leq b c(G) b c(H)$. Watts [18] observed that this inequality can be strict. However, equality always holds for the fractional analogue, given in Theorem 5.3. That is, $b c^{*}(G \tilde{\times} H)=b c^{*}(G) b c^{*}(H)$. The same can be said for the weak product. Watts [19] observed that $b c(G \times H) \leq 2 b c(G) b c(H)$ and that equality need not hold, while equality holds in the corresponding fractional analogue, also given in Theorem 5.3. The proofs of Lemma 5.2 and Theorem 5.3 first appeared in [19].

Lemma 5.2 1. Let $G$ and $H$ be bipartite graphs with ordered bipartitions $\left(X_{G}, Y_{G}\right)$ and $\left(X_{H}, Y_{H}\right)$ respectively. Each ordered biclique of $G \tilde{\times} H$ is a subgraph of an ordered product biclique of $G \tilde{\times} H$.

2. Let $G$ and $H$ be graphs. Each biclique of $G \times H$ is a subgraph of a product biclique of $G \times H$. 
Proof. The proof of 1 follows below. The proof of 2 is similar and is omitted. Let $K(R, S)$ be a biclique of $G \tilde{\times} H$ with ordered bipartition $R \subseteq X_{G} \times X_{H}$ and $S \subseteq Y_{G} \times Y_{H}$. Let

$$
\begin{aligned}
R_{G} & =\left\{g_{i} \in X_{G}:\left(g_{i}, h_{k}\right) \in R \text { for some } h_{k} \in X_{H}\right\} \\
R_{H} & =\left\{h_{k} \in X_{H}:\left(g_{i}, h_{k}\right) \in R \text { for some } g_{i} \in X_{G}\right\} \\
S_{G} & =\left\{g_{j} \in Y_{G}:\left(g_{j}, h_{\ell}\right) \in S \text { for some } h_{\ell} \in Y_{H}\right\} \\
S_{H} & =\left\{h_{\ell} \in Y_{H}:\left(g_{j}, h_{\ell}\right) \in S \text { for some } g_{j} \in Y_{G}\right\} .
\end{aligned}
$$

Then $R \subseteq R_{G} \times R_{H} \subseteq X_{G} \times X_{H}$ and $S \subseteq S_{G} \times S_{H} \subseteq Y_{G} \times Y_{H}$. Each vertex of $R_{G}$ is adjacent to each vertex of $S_{G}$, so $K\left(R_{G}, S_{G}\right)$ is an ordered biclique of $G$. Similarly, $K\left(R_{H}, S_{H}\right)$ is an ordered biclique of $H$. Consequently, $K(R, S)$ is a subgraph of the ordered product biclique $K\left(R_{G}, S_{G}\right) \tilde{\times} K\left(R_{H}, S_{H}\right)=K\left(R_{G} \times R_{H}, S_{G} \times S_{H}\right)$.

Theorem 5.3 1. Let $G_{i}, 1 \leq i \leq k$, be bipartite graphs with ordered bipartitions $\left(X_{G_{i}}, Y_{G_{i}}\right)$ respectively. Then $b c^{*}\left(G_{1} \tilde{\times} \cdots \tilde{\times} G_{k}\right)=\prod_{i=1}^{k} b c^{*}\left(G_{i}\right)$.

2. Let $G_{i}, 1 \leq i \leq k$, be graphs. Then $b c^{*}\left(G_{1} \times \cdots \times G_{k}\right)=2^{k-1} \prod_{i=1}^{k} b c^{*}\left(G_{i}\right)$.

Proof. The proof of 1 follows below. The proof of 2 is similar and is omitted. It suffices to prove the theorem for $k=2$; the general case follows directly by induction on $k$. Let $G=G_{1}$ and $H=G_{2}$. The proof that $b c^{*}(G \tilde{\times} H) \leq b c^{*}(G) b c^{*}(H)$ is similar to the proof of Theorem 5.1 and is omitted.

To show the reverse inequality, let $v_{G}$ and $v_{H}$ be optimal weightings of the edges of $G$ and $H$, respectively, for (2). For each edge $e=\left(g_{i}, h_{k}\right)\left(g_{j}, h_{\ell}\right)$ of $G \tilde{\times} H$ with $\left(g_{i}, h_{k}\right) \in$ $X_{G} \times X_{H}$ and $\left(g_{j}, h_{\ell}\right) \in Y_{G} \times Y_{H}$, construct a weighting $v$ of the edges of $G \tilde{\times} H$ with $v(e)=v_{G}\left(g_{i} g_{j}\right) v_{H}\left(h_{k} h_{\ell}\right)$.

Note that by construction $v(e) \geq 0$ for each edge $e$ of $G \tilde{\times} H$. Let $B=K(R, S)$ be an ordered biclique of $G \tilde{\times} H$ with $R \subseteq X_{G} \times X_{H}$ and $S \subseteq Y_{G} \times Y_{H}$. By Lemma 5.2 $B$ is a subgraph of an ordered product biclique of $G \tilde{\times} H$. That is, $B$ is a subgraph of an ordered product biclique $B_{G} \tilde{\times} B_{H}$ for some $B_{G} \in \mathcal{B}(G)$ and $B_{H} \in \mathcal{B}(H)$. Note that $e=\left(g_{i}, h_{k}\right)\left(g_{j}, h_{\ell}\right)$ is an edge of $B_{G} \tilde{\times} B_{H}$ if and only if $g_{i} g_{j}$ and $h_{k} h_{\ell}$ are edges of $B_{G}$ and $B_{H}$, respectively. Then for each such ordered biclique $B$,

$$
\begin{aligned}
& \sum_{e \in B} v(e) \leq \sum_{e \in B_{G} \tilde{\times} B_{H}} v(e) \\
& =\sum_{\left(g_{i}, h_{k}\right)\left(g_{j}, h_{\ell}\right) \in B_{G} \tilde{\times} B_{H}} v_{G}\left(g_{i} g_{j}\right) v_{H}\left(h_{k} h_{\ell}\right) \\
& =\sum_{\substack{g_{i} g_{j} \in B_{G} \\
h_{k} h_{\ell} \in B_{H}}} v_{G}\left(g_{i} g_{j}\right) v_{G}\left(h_{k} h_{\ell}\right) \\
& =\left(\sum_{e \in B_{G}} v_{G}(e)\right)\left(\sum_{e \in B_{H}} v_{H}(e)\right) \\
& \leq 1 \text {. }
\end{aligned}
$$


Therefore, $v$ satisfies the constraints of (2) and it follows that

$$
\begin{aligned}
& b c^{*}(G \tilde{\times} H) \geq \sum_{e \in E(G \tilde{\times} H)} v(e) \\
& =\sum_{\left(g_{i}, h_{k}\right)\left(g_{j}, h_{\ell}\right) \in E(G \tilde{\times} H)} v_{G}\left(g_{i} g_{j}\right) v_{H}\left(h_{k} h_{\ell}\right) \\
& =\sum_{\substack{g_{i} g_{j} \in E(G) \\
h_{k} h_{\ell} \in E(H)}} v_{G}\left(g_{i} g_{j}\right) v_{H}\left(h_{k} h_{\ell}\right) \\
& =\left(\sum_{e \in E(G)} v_{G}(e)\right)\left(\sum_{e \in E(H)} v_{H}(e)\right) \\
& =b c^{*}(G) b c^{*}(H) \text {. }
\end{aligned}
$$

Consequently, $b c^{*}(G \tilde{\times} H)=b c^{*}(G) b c^{*}(H)$.

Acknowledgements: The author is grateful to the referees for several suggestions and comments which led to the overall improvement of this article. The author is also grateful to D. Gregory for mentioning the definition of $b p^{*}(G)$, observing that edges in the same orbit of Aut $G$ may be assigned the same weight, and for suggesting Theorem 3.2.

\section{References}

[1] C. Berge, Fractional Graph Theory, ISI Lecture Notes 1 (Macmillan of India, 1978).

[2] J. A. Bondy and U. S. R. Murty, Graph Theory with Applications (North Holland, New York, 1976).

[3] E.D. Boyer and B.L. Shader, On biclique decompositions of complete $t$-partite graphs, Linear Algebra Appl. 217 (1995) 31-40.

[4] V. Chvátal, Linear Programming (W. H. Freeman and Company, New York, 1983).

[5] D. de Caen, D. A. Gregory, T. D. Henson, J. R. Lundgren and J. S. Maybee, Real rank as a bound for non-negative integer rank, J. Comb. Math. and Comb. Comp. 11 (1992) 47-53.

[6] D. de Caen, D. A. Gregory and N. J. Pullman, The boolean rank of zero-one matrices I, in: Proc. Third Caribbean Conference on Combinatorics and Computing (Barbados 1981) 169-173.

[7] D. de Caen, D. A. Gregory and N. J. Pullman, The boolean rank of zero-one matrices II, in: Proc. Fifth Caribbean Conference on Cominatorics and Computing (Barbados 1988) 120-126.

[8] F. C. C. Doherty, J. R. Lundgren and D.J. Siewert, Biclique covers and partitions of bipartite graphs and digraphs and related matrix ranks of $\{0,1\}$-matrices, Congressus Numerantium 136 (1999) 73-96. 
[9] R. L. Graham and H. O. Pollak, On the addressing problem for loop switching, Bell System Tech. J. 50 (1971) 2495-2519.

[10] D. A. Gregory and N. J. Pullman, Semiring rank: boolean rank and nonnegative rank factorizations, J. Combin. Inform. System Sci. 8 (1983) 223-233.

[11] D. A. Gregory, B. L. Shader and V. L. Watts, Biclique decompositions and Hermitian rank, Linear Algebra Appl. 292 (1999) 267-280.

[12] F. Harary, D. Hsu and Z. Miller, The biparticity of a graph, J. Graph Theory 1 (1977) no. 2 131-133.

[13] Ki Hang Kim, Boolean Matrix Theory and Applications (Marcel Dekker, Inc., New York, 1982).

[14] T. Kratzke, B. Reznick and D. West, Eigensharp graphs: decomposition into complete bipartite subgraphs, Trans. Amer. Math. Soc. 308 (1988) 637-653.

[15] S. D. Monson, N. J. Pullman and R. Rees, A survey of clique and biclique coverings and factorizations of $(0,1)$-matrices, Bulletin of the I.C.A. 14 (1995) 17-86.

[16] J. Orlin, Contentment in graph theory: covering graphs with cliques, Indag. Math. 39 (1977) 406-424.

[17] E. R. Scheinerman and D. H. Ullman, Fractional Graph Theory: A Rational Approach to the Theory of Graphs (John Wiley and Sons, Inc., New York, 1997).

[18] V. L. Watts, Boolean rank of Kronecker products, Linear Algebra Appl. 336 (2001) 261-264.

[19] V. L. Watts, Biclique covers and partitions of graphs, Ph.d Thesis, Department of Mathematics and Statistics, Queen's Univesity, 2001. 